\title{
Quantification of the ultraviolet radiation (UVR) field in the human eye in vivo using novel instrumentation and the potential benefits of UVR blocking hydrogel contact lens
}

\begin{abstract}
James E Walsh, Jan P G Bergmanson, David Wallace, Gerardo Saldana, Hillary Dempsey, Helen McEvoy, Louis M T Collum
\end{abstract}

\begin{abstract}
Backgroundlaims-Certain degenerative eye conditions occur predominantly nasally, at the limbal region, and are associated with solar ultraviolet radiation (UVR) induced damage. The relative contribution to the in vivo ocular flux of (a) the reflection of UVR incident on the skin of the nose onto the nasal limbus, and (b) the focusing of UVR incident on the temporal side of the cornea onto the nasal limbus were examined.
\end{abstract}

Methods-A novel photodiode sensor array was used to measure the UVR field across the eye. In addition, a novel spectrometer set-up was used to measure the spectrum of radiation refracted across the cornea. The efficacy of UVR blocking hydrogel contact lenses in filtering incident UVR was assessed in vivo.

Results-Qualitative and quantitative data indicated an increase nasally of UVR. Photodiode readings showed a net UVR increase from the temporal to the nasal side. Transmission curves showed that most UVR incident on the limbal region is either absorbed by, or transmitted through, the ocular tissues. This radiation is filtered by UVR blocking soft contact lens.

Conclusions-An increased UVR flux on the nasal side of the eye, due to reflection off the nasal skin, was identified in vivo. Any UVR passing through the cornea is either absorbed by the conjunctiva and/or transmitted through it onto the sclera where it is absorbed. UVR blocking hydrogel contact lenses can eliminate these sources of UVR.

(Br f Ophthalmol 2001;85:1080-1085)

Foundation, The Royal

Victoria Eye and Ear

Hospital, Dublin,

Ireland

D Wallace

H Dempsey

L M T Collum

Correspondence to:

Dr James E Walsh, School of Physics, Dublin Institute of

Technology, Kevin Street,

Dublin 8, Ireland

james.walsh@dit.ie

Accepted for publication

7 February 2001 partial closing of the eyelids in response to high visible light levels. These are only partially effective and incident UVR absorbed and integrated over long periods may cause degenerative effects. ${ }^{8-15}$ Methods to lower incident UVR include covering, filtering, and shading. ${ }^{8}$ In particular, sunglasses and headwear that shade the eyes from direct visible and UVR insolation do not generally provide complete protection from scattered and temporally incident light.

There is epidemiological evidence that photokeratitis, climatic droplet keratopathy, xeroderma pigmentosum, pingueculae, cortical cataracts, and pterygium are all examples of acute or degenerative diseases that occur on the ocular surface and that are caused by UVR exposure. ${ }^{16-20}$

Our study was mainly concerned with pterygium and its UVR dependence. Although a pterygium rarely affects vision, it is cosmetically distressing and may require surgery. The efficacy of treatment is reduced by high recurrence rates and significant postoperative complications such as dry eye symptoms, granuloma, and corneal scarring. ${ }^{21}$ The incidence of pterygia, particularly in regions near the equator, approaches $10 \% .{ }^{22}{ }^{23}$ Epidemiological evidence indicates that UVR is a significant risk factor in the development of pterygium. ${ }^{24-27}$

Histopathologically, a pterygium involves basophilic degeneration of the subepithelial stroma in the bulbar region of the conjunctiva. ${ }^{28}{ }^{29}$ New evidence also implicates deficient stem cells, which may promote corneal invasion. ${ }^{30}$ Pterygium predominantly occurs on the nasal side of the eye and Coroneo et $\mathrm{l}^{31}{ }^{32}$ proposed that tangentially incident UVR focused onto the nasal limbus was the main causative factor. Owing to its short wavelength and high energy, UVR may initiate photochemical reactions leading to tissue changes predisposing to pterygium formation. ${ }^{14}$

Several other predisposing factors have been suggested including a genetic predisposition, chronic dryness, heat, and irritation. ${ }^{33}$ The latter theories are not supported by the fact that pterygia are highly prevalent in Eskimos, surfers, and sailors. ${ }^{31}$ A notable consistent predisposing factor is terrain reflectivity. ${ }^{14}{ }^{31}$ An Eskimo and an Australian aborigine are the only documented patients ever blinded by pterygia. $^{3435}$ This supports the UVR theory because of increased light entering the eye from above and below from high UVR albedo.

A second theory explaining the nasal predilection of pterygia is that UVR is reflected off the skin of the nose and adjacent facial regions 


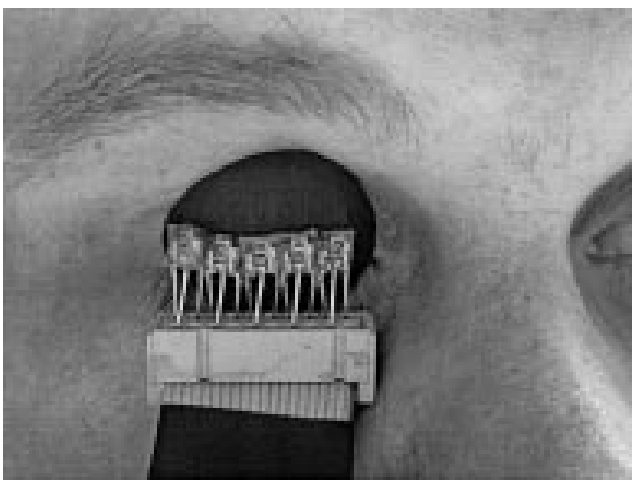

Figure 1 UVR photodiode sensor array mounted over left eye with sensor number one on the temporal side and sensor five on the nasal side.

onto the nasal side of the eye. Both nasal reflection and temporally incident light refracted across the cornea ${ }^{36}$ can still cause UVR exposure in the presence of sunglasses or eye shading headwear.

However, neither of these two hypotheses has been previously investigated in vivo across the UVR waveband. The aims of the present study were the design, construction, and testing of novel sensor systems to confirm the relative contribution of these two sources of increased nasal UVR exposure, which are potential causes of ocular diseases such as pterygium. Also, the efficacy of UVR blocking hydrogel contact lenses in filtering incident UVR was assessed in vivo.

\section{Materials and methods}

To test the reflective theory in vivo an array of UVR sensors was built to sample the incoming light field at or near the exposed ocular surface. For the refractive theory a fibre optic spectrophotometer based sensor was placed at the nasal conjunctival limbus to detect any light being refracted across the cornea and determine its spectrum. These two unique experimental set-ups are now described.

REFLECTIVE SENSOR ARRAY

The primary components on the reflective sensor array were five Texas Instruments TSL250 photodiodes. These light detectors have a spectral response curve which extends down to $300 \mathrm{~nm}$ in the UVR region of the spectrum, covering all of the UVA region and part of the UVB region. They have a field of view of approximately plus or minus 60 degrees from the normal and therefore photons incident over a broad range of angles can be detected. High sensitivity and integral amplifiers provide high signal output allowing low levels of UVR to be detected. Each sensor package is $5 \mathrm{~mm}$ square so five of them mounted side by side will cover a region about $25 \mathrm{~mm}$, which covers the average horizontal diameter of exposed ocular tissue. The five sensors were mounted on a plastic shell, normally placed on the eye to protect it during eyelid surgery, with sensors number one and two on the temporal side of the pupil, sensor three directly over the pupil and sensors four and five on the nasal side, as shown in Figure 1.
Analogue voltages output from the sensor are digitised and stored in a laptop computer, using the LABVIEW program, and all five sensors are read out in milliseconds, greatly reducing any unwanted signal variations across the sensor caused by movement of the test subject. Relative readings across the sensor are calculated by recording the signal from each sensor in a uniform light field with no subject present and dividing these values into the respective signals when the subject is in place. To provide a stable test environment a light box, illuminated by a stable, diffuse UV visible-NIR tungsten light source, was constructed. As the interior walls of the box were white, the test subject is therefore in a relatively uniform diffuse light field, which approximates the diffuse solar light field. A low pass filter in front of the light source was used to restrict the light to the spectral region of interest.

\section{REFRACTIVE SENSOR}

The principal components of the refractive sensor set-up are shown in Figure 2. A modified slit lamp is used to mount two fibreoptics at the cornea. The input fibre images light from a UV-visible-NIR tungsten light source onto the cornea, at an angle of about 30 degrees posterior to the coronal plane. The output fibre detects the light emerging from the cornea or reflected off the sclera, depending on the relative position of the eye. The detected light is presented to an Ocean Optics S2000 fibreoptic spectrometer where it is dispersed onto a detector array whose signal is read into and digitised by a laptop computer. Transmission spectra are computed using the spectrum of the light source as a reference, which is recorded when no test subject is present. Each spectrum can be recorded in about $20 \mathrm{~ms}$, greatly reducing any unwanted signal variations caused by movement of the test subject.

To block the UVR that is focused across the eye, two different UVR blocking contact lenses were used; the Acuvue 2 (Etafilcon, 58\% water content, Vistakon) and the Precision UV (Vasurfilcon, $74 \%$ water content, Wesley Jessen). Near plano lens power was selected to minimise the refractive effects of the lenses.

\section{Results}

Test measurements with the reflective sensor array were made on a white polystyrene manikin head to determine the repeatability of the set-up. Sensor readings were taken in the light box with no head present and with the head present and the relative change in intensity calculated. Figure $3 \mathrm{~A}$ shows the relative change in intensity for the five sensors across the dummy head averaged over a number of separate experiments. All the sensors show an increase, when the head is present, over the ambient light signal. Using the dummy head there is a significantly increased signal in sensor number five, which is on the nasal side, relative to the other four sensors, indicating increased light scattered and reflected onto it from the facial structure on the nasal side. Similar test 


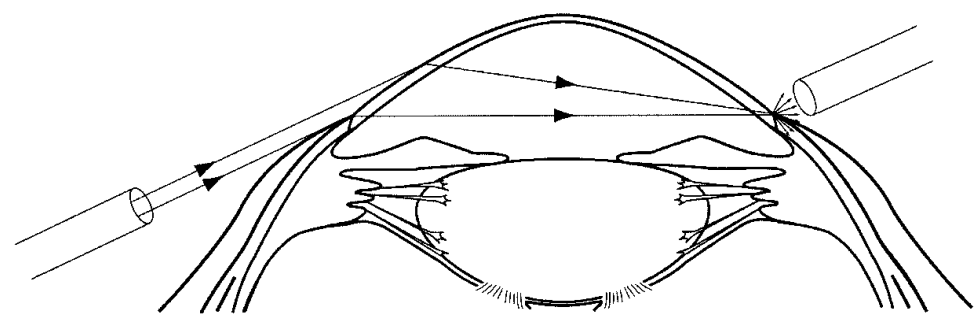

Figure 2 Schematic diagram of the refractive sensor set-up. Light from a fibreoptic illuminates the cornea temporally and is refracted across to a sensing fibre optic.

measurements were then made on 12 test subjects and in 10 of the 12 , intensity at the nasal sensor is significantly higher than that at the lateral sensor. Measurements on each test subject were carried out five times and error bars computed from the standard deviation about the mean of the five samples for each sensor $(\mathrm{p}<0.05$, sensor 1 versus sensor 5, paired Student's $t$ test for the subjects shown in Fig 3). Typical data from the population sampled are presented in Figures 3B-F). The data for test subjects 1 to 3, shown in Figures 3B-D, show the same type of nasal increase as the test head, this was typical for most subjects tested.
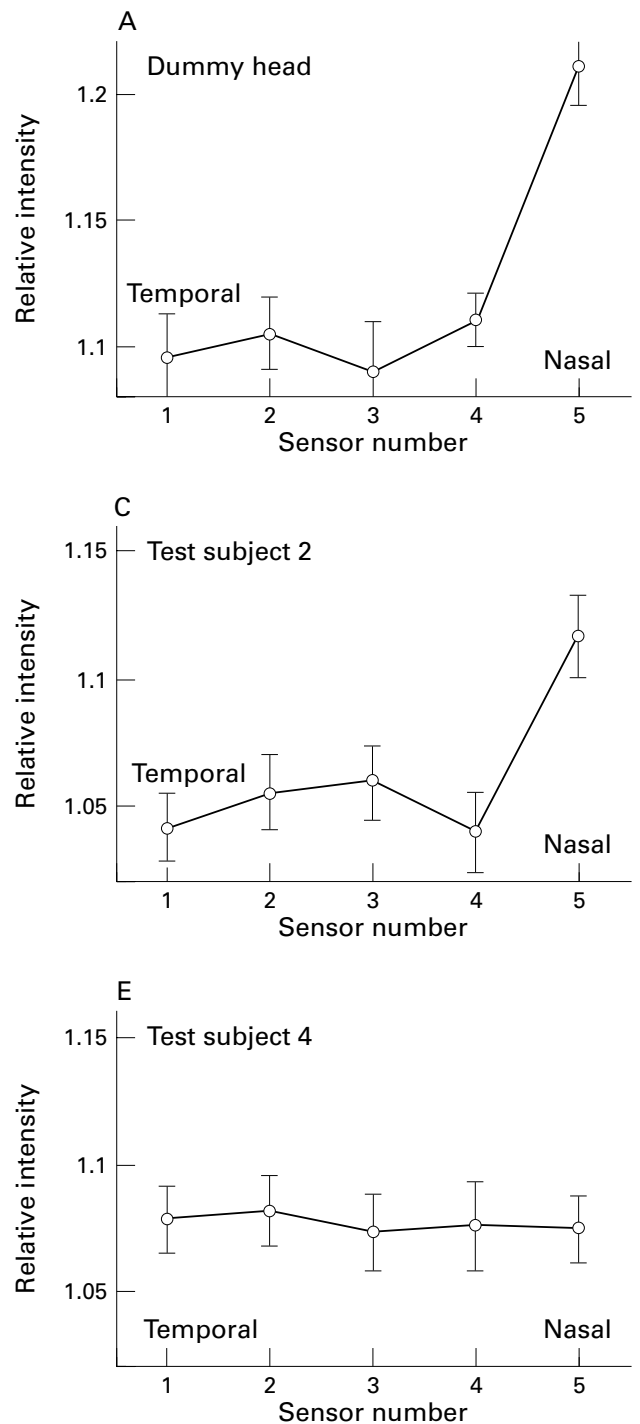

Whereas the data for test subject 4 , shown in Figure $3 \mathrm{E}$, shows a flat response and that for test subject 5, shown in Figure 3F, shows a decrease on the nasal side.

Typical qualitative data from the refractive sensor set-up are shown in the digital image in Figure 4 (left). UV-visible-NIR light is imaged onto the eye from the fibreoptic illuminator on the left, refracted across the eye by the cornea. In this image the illumination angle is roughly 10 degrees posterior to the coronal plane so the light emerges from the nasal side of the cornea allowing us to measure its transmission in vivo. Figure 4 (right) shows light imaged at roughly 30 degrees posterior to the coronal plane and imaged, across the cornea, onto the conjunctiva at the nasal limbus. A white spot is visible where the light reflects off the ocular tissue. The transmission spectrum of the conjunctiva, in vivo, can be calculated by comparing the spectrum of this spot with that of the original light source. The transmission spectrum of the cornea can be calculated, in vivo, by comparing the spectrum of light passing through the cornea with that of the original light source. The spectrum of light passing through the cornea
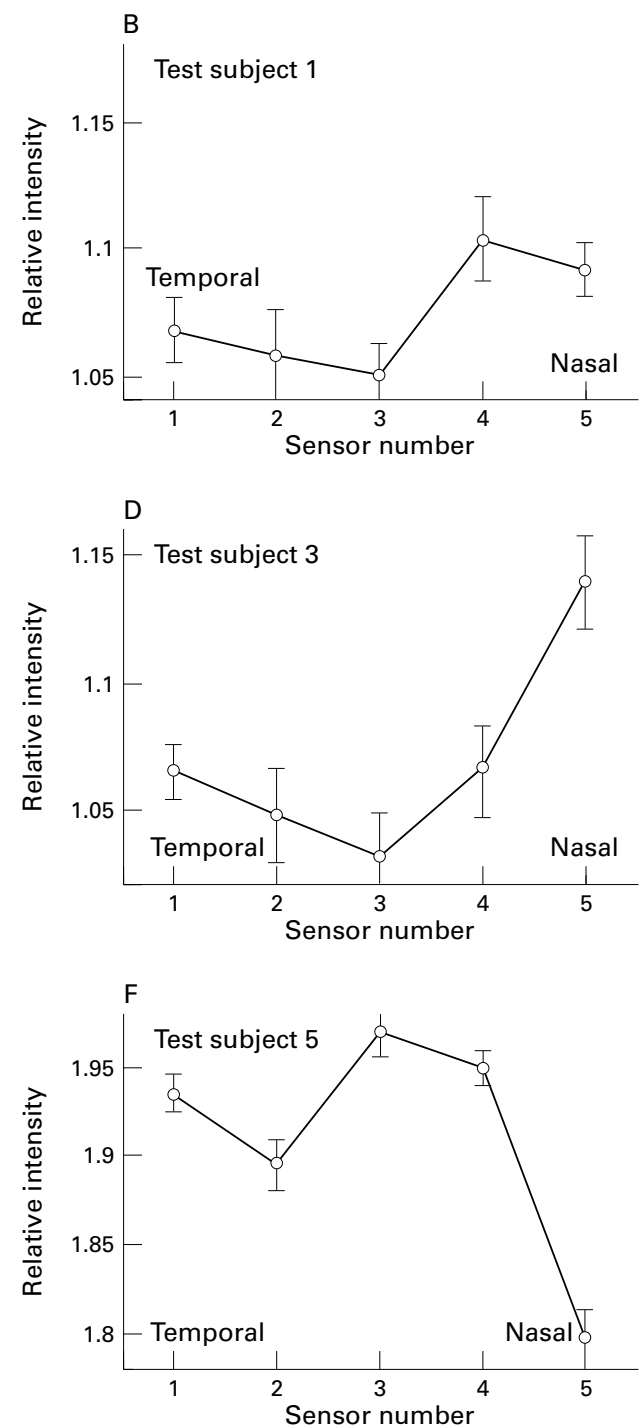

Figure 3 The relative change in intensity for the five sensors across the dummy head eye and five test subjects. 

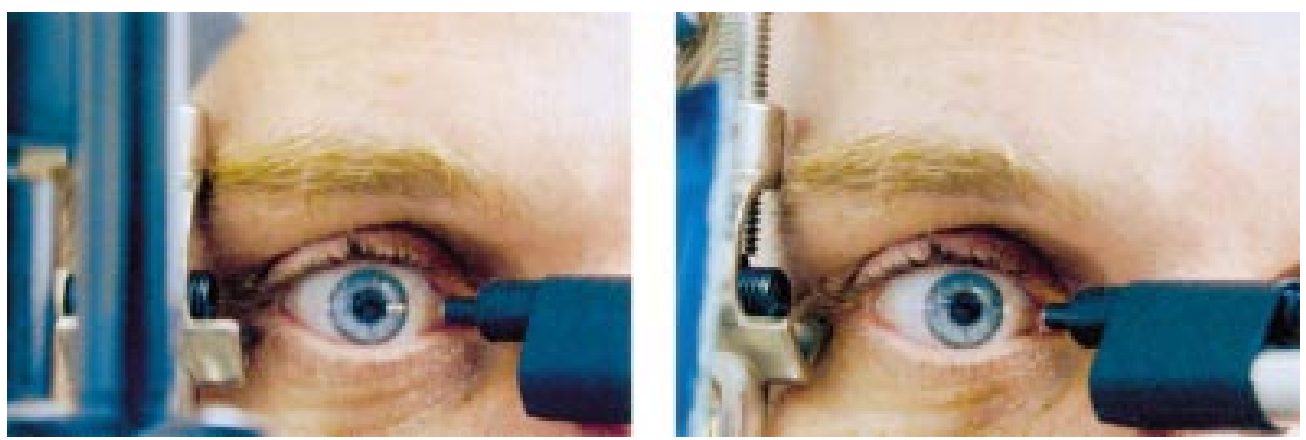

Figure 4 (Left) Typical qualitative data from the refractive sensor set-up showing light refracted across the cornea from the temporal side and emerging from it nasally. (Right) Typical qualitative data from the refractive sensor set-up showing light refracted across the cornea from the temporal side and imaged onto the limbal, conjunctival, and scleral junction.

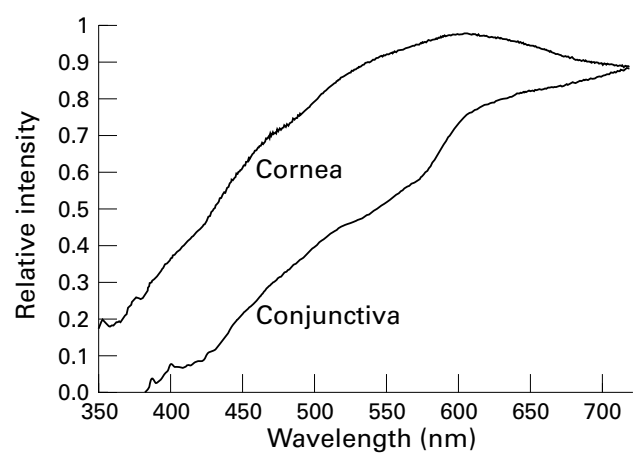

Figure 5 Typical transmission spectra of the cornea and conjunctiva measured, in vivo, with the refractive set-up.

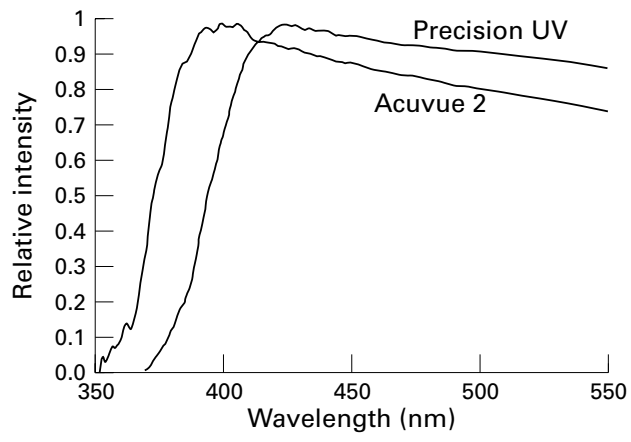

Figure 6 The transmission spectra of two UVR blocking hydrogel contact lenses, the Acuvue 2 and the Precision UV, measured in vivo.

can be measured by coupling it into the detection fibre before it is imaged onto the conjunctiva. Figure 5 shows typical transmission spectra of the cornea and conjunctiva measured, in vivo, with the refractive set-up. Note that light in the UVR region emerges from the cornea but is absorbed in the conjunctiva indicating that any UVR incident temporally on the eye is focused onto the nasal conjunctiva.

If UVR blocking hydrogel contact lenses are placed on the cornea the transmission spectrum of the cornea can again be measured and compared with that shown in Figure 5. Figure 6 shows the transmission spectra of the two UVR blocking hydrogel contact lenses used, the Acuvue 2 and the Precision UV, measured in vivo. These spectra correlate well with the data published by the manufacturers and others $^{3738}$ and show that both lens types prevent unwanted UVR from being refracted across the eye onto the nasal limbus. A comparison between the two contact lenses shows that the UVR cut off for the Precision UV lenses is higher up the wavelength scale than the Acuvue 2, thus affording greater UVR protection.

\section{Discussion}

Under controlled lighting conditions the present study demonstrated in vivo that temporally incident UVR is both reflected and refracted to a focus near the nasal limbus. Previous studies used visible light to illustrate tangential corneal refraction. ${ }^{3132} 36$ To our knowledge, the literature contains no previous quantitative reports on UVR in vivo reflection off the skin on the nose and adjacent facial features. For most subjects the facial features interact to create an area of enhanced light intensity and this area coincided with the medial aspect of the eye. However, this medial area of enhanced UVR intensity is not common to all individuals. For example, subject 5 in this study showed reduced intensity on the nasal side but had no apparent differences in visible skin tone, indicating that, within the study group, facial structure and skin tone were not the defining factors. It should be noted that the sample group tested consisted of both male and female fair skinned subjects, with no obvious differences in facial structure.

The refractive instrumentation set-up in the present study permitted the in vivo transmission curves for both cornea and conjunctiva to be established and the assessment of the relative UVR blocking efficacy of two UVR blocking contact lenses. Data from the unique in vivo optical set-up were validated by the fact that the transmission curves measured for the UVR blocking contact lenses were similar to previously published curves. ${ }^{37} 38$ These lenses, which cover the cornea limbus to limbus, provide unique protection from tangentially incident UVR and daytime lens wear affords the user continuous protection. Perhaps our current view of the hydrogel contact lens option as UVR protection should be upgraded for people working in locations of high UVR intensity. ${ }^{38}$

The maximum relative variation recorded across the reflective sensor array was about $10 \%$ so the overall instantaneous effect was not great. Likewise, the UVR increase at the nasal limbus as a result of the tangentially focused UVR has been modelled by Coroneo to be up to a factor of $20^{31}$ but only about $20 \%$ of the 
incident UVR emerges from the cornea to irradiate the tissues of the nasal limbus. However, both these mechanisms for increased UVR have a cumulative dosimetric effect, which could explain the high incidence of pterygium in the aged population. Absolute measurements carried out under natural solar UVR conditions, using both methods, will yield further information and allow us to assess the effects of different ground reflectance on the data.

Based on our studies and the existing literature, tangentially focused UVR had greater effect than the nasally reflected UVR. ${ }^{31}{ }^{32}$ Radiation crossing the cornea tangentially and focused at the nasal limbus will, at this medial point, travel through the cornea a second time in a posterior to anterior direction. Thus, the corneal structure most sensitive to UVR, the epithelium, is reached last if the radiation is not already absorbed. The first epithelial cells irradiated in this manner are the basal cells among which the stem cells are located. Peripherally located stem cells participate in central corneal epithelial renewal at all times. ${ }^{39}{ }^{40}$ These cells, vital to the cornea, are sensitive and their proliferation is easily inhibited. ${ }^{41}{ }^{42}$ UVR induced stem cell loss may result in a stem cell deficiency, which has been proposed as an underlying cause of corneal invasion by pterygium. ${ }^{30}$

We have shown, for the first time in human subjects, that tangentially incident UVR on the temporal limbus is focused on the opposite nasal limbus in vivo. The exact role of UVR in the aetiology of pterygium, and the relative contribution of the mechanisms discussed, have yet to be established. However, of all factors studied, a UVR association with pterygium formation appears to be the most feasible. ${ }^{931}$ We currently lack alternative conclusive explanations for the nasal bias of pterygia. Pterygium is generally defined as a degeneration and characterised histopathologically as a subepithelial stromal basophilic degeneration of collagen and elastic tissue. ${ }^{28-30}$ Indeed, Apple and $\mathrm{Rabb}^{28}$ state that these changes are similar to that observed in solar keratosis, a skin condition observed in individuals who received a high lifetime UVR dose.

In addition, we currently lack effective treatment for pterygia and surgery often leads to many functional and cosmetic complications and frequent recurrences. ${ }^{21}$ Given the potential corneal involvement and unappealing cosmetic effect of pterygia, preventing UVR insolation of the ocular tissues is arguably our best option.

In summary, qualitative and quantitative data indicating an increase in the UVR flux on the nasal limbus, by two mechanisms, has been shown. For light reflected on to the ocular region, the majority of test subjects demonstrated up to $10 \%$ increase in the light field on the nasal side. This nasal bias may be significant when considered dosimetrically over long periods. A concentration of temporally incident UVR, focused across the cornea, has also been shown and the spectral nature of the light incident on the nasal limbus quantified. Data indicate that UVR incident on the nasal conjunctiva is absorbed in the ocular tissue. Both of these mechanisms confirm that there is an increased UVR flux on the nasal side of the eye and this may account for the nasal-temporal bias of certain ocular diseases. Our experiments with contact wear clearly demonstrate that current UVR blocking contact lenses shield the limbal region from direct, reflected, and tangentially refracted UVR. This is a unique benefit provided by the UVR blocking soft contact lens, since spectacles, sunglasses, and shading headwear do not generally offer temporal protection.

This research was funded by Enterprise Ireland, International Collaboration Travel Grant IC99/093, Dublin Institute of Technology Seed Funds 99/02 and 00/26, Wesley Jessen Corporation Travel Grants 1999/2000, and research funds from the Texas Eye Research and Technology Center, University of Houston College of Optometry 1999/00. 1 Gallagher RP, McLean DE, Yang CP, et al. Suntan, sunburn, and pigmentation factors and frequency of acquired melanotic nevi in children. Similarities to melanoma: the

2 Hillenkamp F. Biophysical mechanisms of damage induced by light. In: Cronly-Dillon J, Rosen ES, Marshall J, eds. Hazards of light, myths and realties, eye and skin. New York: Pergamon Press, 1985:21-32.

3 Lindgren G, Diffey BL, Larkö O. Basal cell carcinoma of the eyelids and solar ultraviolet radiation exposure. $\mathrm{Br} F$ Ophthalmol 1998;82:1412-15.

4 Scotto J. Risk factors: solar radiation. In: Harras A ed. Cancer rates and risks. 4th ed. NCI NIH Publ no 96-691, May 1996.

5 Hyde JN. On the influence of light in the production of skin cancer. Am f Med Sci 1906;1311:1-22.

6 Kripke ML. Carcinogenises: ultraviolet radiation. In: FritzKripke $M L$. Carcinogenises: ultraviolet radiation. In: Fritz-
patrick TB, Eisen AZ, Wolff K, Freedberg IM, Austen KF, patrick TB, Eisen AZ, Wolff K, Freedberg IM, Austen KF, eds. Dermatoh

7 Streilen JW, Taylor JR, Vineek V, et al. Relationship between ultraviolet radiation-induced immunosuppression and carcinogenises. F Invest Dermatol 1994;103:107s-11s.

8 Pitts DG, Bergmanson JPG. The UV problem: have the rules changed? F Am Optom Assoc 1989;60:420-4.

9 Bergmanson JPG, Soderberg PG. The significance of ultraviolet radiation for eye diseases:a review with comments on the efficacy of UV-blocking contact lenses. Ophthalmic Physiol Opt 1995;15:83-91.

10 Cullen AP, Perera SC. Sunlight and human conjunctival action spectrum. Ultraviolet radiation hazards. SPIE Proc 1994;2134:24-30

11 Pitts DG. Principles in ocular protection. In: Pitts DG, Kleinstein, eds. Environmental vision. Boston: ButterworthHeinemann, 1993:259-80.

12 Pitts DG, Bergmanson JPG, Chu LWF, et al. Ultrastructural analysis of corneal exposure to UV radiation. Acta Ophthalmol 1987;65:263-73.

13 Pitts DG, Cullen AP, Hacker PD. Ocular effects of ultraviolet radiation 295 to $365 \mathrm{~nm}$. Invest Ophthalmol Vis Sci 1977:932-9.

14 Sliney DH. The focusing of ultraviolet radiation in the eye and ocular exposure. In:Taylor HR, ed. Pterygium. The Hague: Kugler, 2000:29-40.

15 Taylor HR, West SK, Munoz B, et al. The long-term effects of visible light on the eye. Arch Ophthalmol 1992;110:99104.

16 Al-Rajhi AA, Cameron JA. Recurrence of climatic droplet keratopathy. Acta Ophthalmol Scand 1996;74:642-4.

17 Bergmanson JPG. Corneal damage in photokeratitis-why is it so painful? Optom Vis Sci 1990;67:407-13.

18 Cogan DG, Kinsey VE. Action spectrum for keratitis produced by ultraviolet radiation. Arch Ophthalmol 1946; produced

19 Freedman A. Labrador keratopathy. Arch Ophthalmol 1965; $74: 198-202$

20 Zuclich JA. Ultraviolet induced photochemical damage in ocular tissue. Health Physics 1989;56:671-82.

1 Snibson GR, Luu CD, Taylor HR. Pterygium surgery in Victoria: a survey of ophthalmologists. Aust NZ $\mathcal{F}$ Ophthalmol 1998;26:271-6.

22 Hirst LW. Distribution, risk factors, and epidemiolgy of pterygium. In: Taylor HR, ed. Pterygium. The Hague: Kugler, 2000:29-40.

23 Panchapakesan L, Haurihan F, Mitchell P. Prevalence of pterygium and pinguecula: the Blue Mountains Eye Study. Aust NZ F Ophthalmol 1998;26:52-5.

24 Mackenzie FD, Hirst LW, Battistutta D, et al. Risk analysis in the development of pterygia. Ophthalmology 1992;99: 1056-61. 
25 Taylor HR, Wesk SK, Rosenthal FS, et al. Corneal changes associated with chronic UV irradiation. Arch Ophthalmol $1989 \cdot 107: 1481-4$

26 Threlfall TJ, English DR. Sun exposure and pterygium of the eye: a dose-response curve. Am $\mathcal{F}$ Ophthalmol 1999;128 $280-7$

27 McCarty CA, Fu CL, Taylor HR. Epidemiology of pterygium in Victoria, Australia. Br f Ophthalmol 2000;84: 289-92.

28 Apple DJ, Rabb MF. Conjunctiva and eyelids. In: Ocular pathology: clinical applications and self-assessment. 5th ed. S Louis: Mosby, 1998:563-604

29 Yanoff M, Fine BS. Conjunctiva. In: Ocular pathology. 4th ed. Spain: Mosby-Wolfe, 1996:205-29.

30 Tseng SCG, Lee SB, Li DQ. Limbal stem cell deficiency in the pathenogenesis of pterygium. In: Taylor HR, ed. Pterygium. The Hague: Kugler, 2000:41-55.

31 Coroneo MT Pterygium as an early indicator of ultraviolet Corone MT. Pterygium as an early indicator of ultraviolet mol 1993;77:734-9.

32 Coroneo MT, Müller-Stolzenburg NW, Ho A. Peripheral light focusing by anterior eye and the ophthalmohelioses. Ophthalmic Surg 1991;22:705-11.

33 Widmark EJ. Über die Durchdringlichkeit der Augenmedien für ultraviolette strahlen. Scand Arch Physiol 1892;3: $14-46$
34 Taylor HR, Hollows FC. Pterygium leading to blindness: a case report. Aust F Ophthalmol 1978;6:155-6.

35 Fritz $\mathrm{MH}$. Blindness from multiple pterygiums in an Alaskan native. Am $\mathcal{f}$ Ophthalmol 1955;39:572.

36 Cullen AP, Oriowo OM, Viosin AC. Anterior eye focusing of ultraviolet and visible radiation albedo. Clin Exp Optom 1997;80:80-6.

37 Harris MG, Haririfar M, Hirano KY. Transmittance of tinted and UV-blocking disposable contact lenses. Optom Vis Sci 1999;76:177-80.

38 Pitts DG, Lattimore MR. Protection against UVR using Vistakon UV-blocking soft contact lens. Int Contact Lens Clin 1987;14:22-30.

39 Kruse FE. Stem cells and corneal epithelial regeneration. Eye 1994;8:170-83.

40 Zieske JD. Perpetuation of stem cells in the eye. Eye 1994;8: 163-9.

41 Ladage PM, Yamamoto K, Ren DH, et al. Basal epithelial cell turnover following RGP extended contact lens wear in the rabbit cornea. Invest Ophthalmol Vis Sci 2000;41:S75.

42 Ren DH, Petroll WM, Jester JV, et al. The effect of rigid gas permeable contact lens on proliferation of rabbit corneal and conjunctival epithelial cells. CLAO 1999;25:136-41.

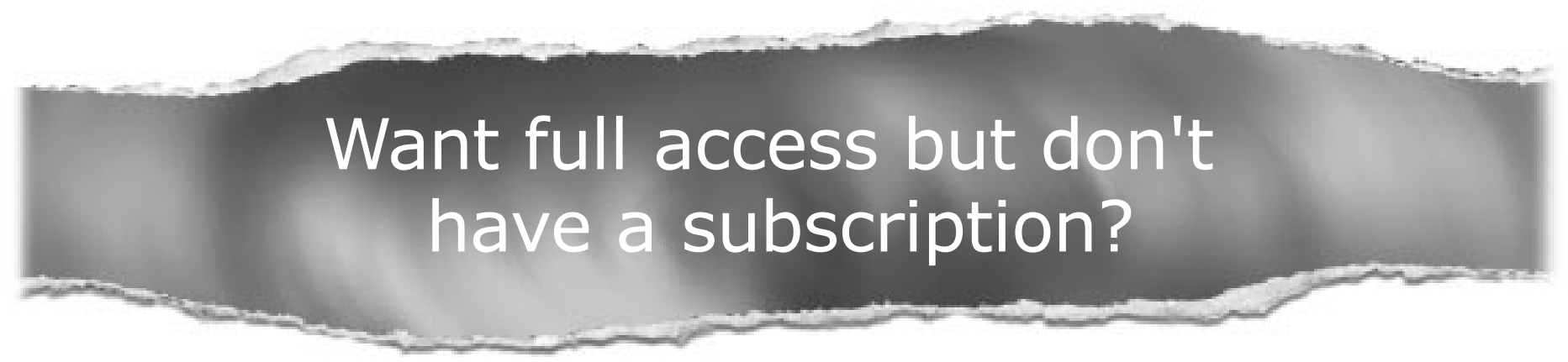

\section{Pay per access}

For just US $\$ 25$ you can have instant access to the whole website for 30 days. During this time you will be able to access the full text for all issues (including supplements) available. You will also be able to download and print any relevant pdf files for personal use, and take advantage of all the special features British Journal of Ophthalmology online has to offer.

www.bjophthalmol.com 